\section{(C) OPEN ACCESS}

\title{
Beyond motor neurons: expanding the clinical spectrum in Kennedy's disease
}

\author{
Raquel Manzano, ${ }^{1}$ Gianni Sorarú, ${ }^{2}$ Christopher Grunseich, ${ }^{3}$ Pietro Fratta, ${ }^{4}$ \\ Emanuela Zuccaro, ${ }^{5}$ Maria Pennuto, ${ }^{5,6}$ Carlo Rinaldi $^{1}$
}

${ }^{1}$ Department of Physiology, Anatomy and Genetics, University of Oxford, Oxford, UK ${ }^{2}$ Department of Neurosciences, Neuromuscular Center, University of Padova, Padova, Italy

${ }^{3}$ Neurogenetics Branch, National Institute of Neurological Disorders and Stroke, National Institutes of Health, Bethesda, Maryland, USA

${ }^{4}$ Department of Motor Neuroscience and Movement Disorders, UCL Institute of Neurology, London, UK ${ }^{5}$ Department of Biomedical Sciences, University of Padova, Padova, Italy

${ }^{6}$ Dulbecco Telethon Institute, Centre for Integrative Biology, University of Trento, Trento, Italy

Correspondence to Dr Carlo Rinaldi, Department of Physiology, Anatomy and Genetics, University of Oxford, Oxford OX1 3QX, UK; carlo. rinaldi@dpag.ox.ac.uk

Received 26 October 2017 Revised 21 December 2017 Accepted 21 December 2017 Published Online First 20 January 2018

Check for updates

To cite: Manzano $\mathrm{R}$ Sorarú G, Grunseich C, et al. J Neurol Neurosurg Psychiatry 2018:89:808-812.

\section{ABSTRACT}

Kennedy's disease, or spinal and bulbar muscular atrophy (SBMA), is an X-linked neuromuscular condition clinically characterised by weakness, atrophy and fasciculations of the limb and bulbar muscles, as a result of lower motor neuron degeneration. The disease is caused by an abnormally expanded triplet repeat expansions in the ubiquitously expressed androgen receptor gene, through mechanisms which are not entirely elucidated. Over the years studies from both humans and animal models have highlighted the involvement of cell populations other than motor neurons in SBMA, widening the disease phenotype. The most compelling aspect of these findings is their potential for therapeutic impact: muscle, for example, which is primarily affected in the disease, has been recently shown to represent a valid alternative target for therapy to motor neurons. In this review, we discuss the emerging study of the extra-motor neuron involvement in SBMA, which, besides increasingly pointing towards a multidisciplinary approach for affected patients, deepens our understanding of the pathogenic mechanisms and holds potential for providing new therapeutic targets for this disease.

\section{INTRODUCTION}

Spinal and bulbar muscular atrophy (SBMA) is an $\mathrm{X}$-linked neuromuscular condition caused by expansions of a CAG repeat in the androgen receptor (AR) gene. ${ }^{1}$ Disease onset ranges from about 18 to 64 ages, with affected males mostly presenting in the fourth decade of life with tremor, cramping, proximal and distal weakness, and muscle atrophy, secondary to lower motor neuron degeneration. ${ }^{2}$ Involvement of the bulbar muscles is a frequent finding, accounting for dysarthria and dysphagia, hypernasality and perioral fasciculations. Fatigue when chewing and occasionally jaw drop are caused by weakness of the temporalis and masseter muscles with selective preservation of pterygoid muscles. ${ }^{3}$ Affected subjects are at risk of choking on food and aspiration pneumonia because of weakness of the bulbar muscles. ${ }^{4}$ Compared with other motor neuron diseases, such as amyotrophic lateral sclerosis (ALS), disease progression is relatively slow, with muscle strength declining by $2 \%$ per year. ${ }^{5}$ Recent evidence has shown that the muscle atrophy in SBMA is not solely secondary to the motor neuron degeneration but also consequence of a primary myopathic process. In addition, SBMA is frequently complicated by other signs and symptoms such as gynaecomastia, impotence, testicular atrophy and metabolic changes, suggesting a multisystem involvement in the disease (figure 1). These features frequently manifest early in the disease course and can contribute substantially to the morbidity. In this review, we discuss the non-motor neuron abnormalities underlining SBMA symptomatology. We propose that an improved understanding of these features not only could result in better management of the patients SBMA, but also has the potential to shed new light into the disease pathogenesis, lead to the discovery of biomarkers for disease progression and open new treatment avenues.

\section{MECHANISMS OF DISEASE PATHOGENESIS}

The AR is a ligand-dependent, ubiquitously expressed, transcription factor that belongs to the steroid hormone group of nuclear receptors. ${ }^{6}$ Following binding to $5 \alpha$-dihydrotestosterone (DHT) and testosterone, AR dimerizes, undergoes conformational changes and translocates into the nucleus where it recognises androgen response elements in the promoter regions of target genes. ${ }^{6}$ A complete loss of AR function in males results in androgen insensitivity syndrome, in the absence of motor neuron involvement, ${ }^{7}$ favouring a model of toxicity in SBMA, where mechanisms of toxic gain of function and loss of AR activity coexist. The mechanisms of polyQ-AR-mediated toxicity, which are yet to be elucidated, are believed to be partly due to transcriptional dysregulation, potentially resulting in activation of different transcriptional programmes in different subcellular populations. ${ }^{8}$ Additionally, mutant AR is able to self-associate to form soluble oligomers or aggregate into inclusions in a ligand-dependent manner. ${ }^{9}{ }^{10}$ These aggregates locate mostly in the nucleus and may exert toxicity through sequestration of a pool of distinct proteins (ie, heat shock proteins or components of the ubiquitin-proteasome), AR co-activators and other transcription factors, ${ }^{11}$ which is unique to each cell population. These inclusions can be found in neuronal as in non-neuronal tissues and visceral organs of patients with SBMA. ${ }^{12}$ Motor neurons are particularly sensitive to alterations in the proteostasis network, likely due to their extreme polarisation and post-mitotic nature. ${ }^{13}$ On the other side, alterations of protein quality control in muscle, by tilting the balance towards increased protein degradation, are likely the underlying cause of the muscle atrophy observed in this disease. ${ }^{14}$

\section{MUSCLE ATROPHY}

In the last two decades, an increasing number of observations derived from preclinical studies and 


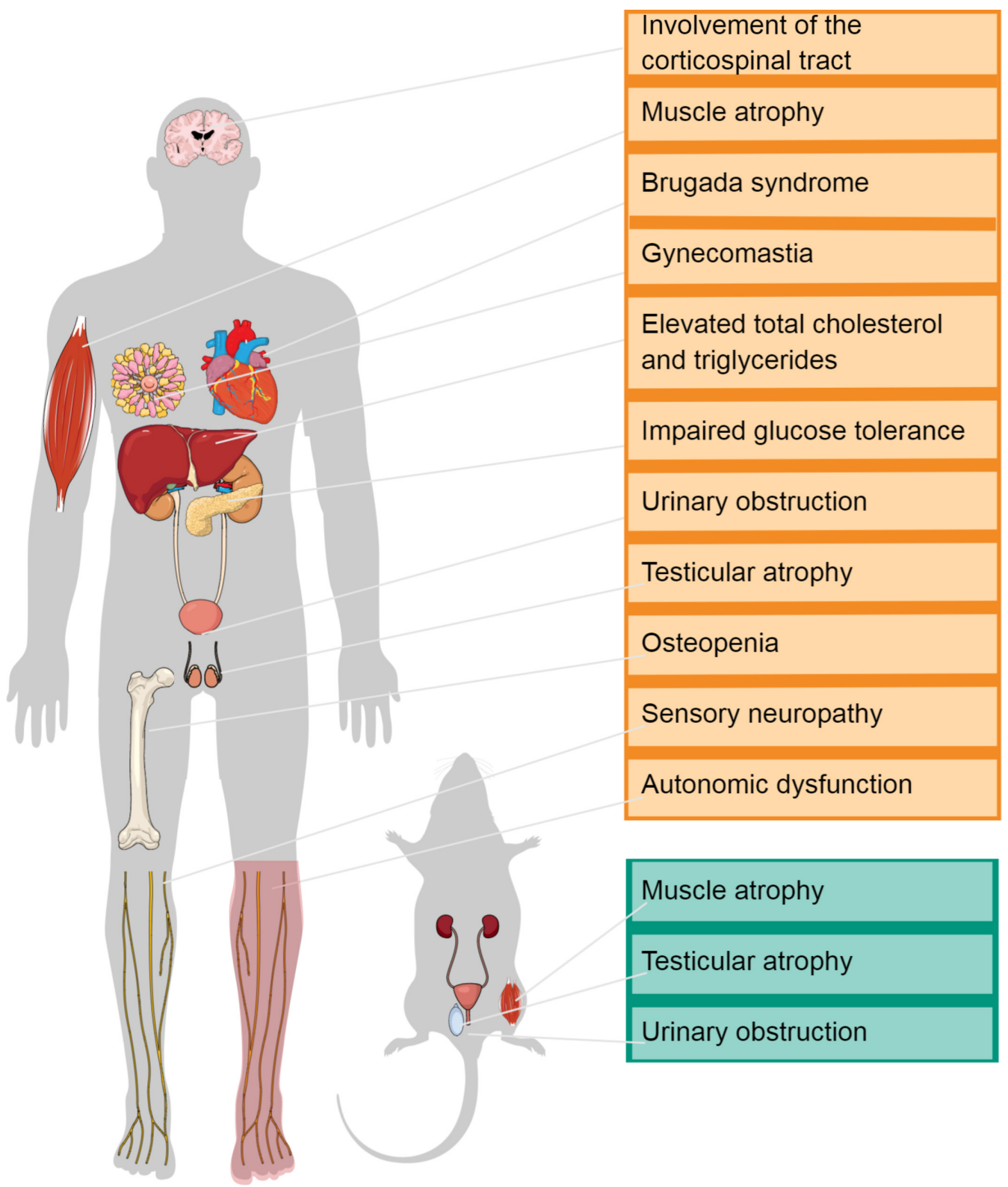

Figure 1 Extra-motor neuron features in spinal and bulbar muscular atrophy.

patient samples have indicated that in SBMA skeletal muscle, rather than solely being a bystander of motor neuron degeneration, is also primarily involved in the disease pathogenesis and represents a target for therapy development. Studies performed on patients with SBMA report mildly elevated levels of serum creatine kinase $(\mathrm{CK})^{15}$ and the increase can be detected prior to the onset of SBMA clinical symptoms. ${ }^{16}$ Histological studies on muscle biopsies have shown the presence of fibre-type grouping, atrophic fibres and angulated fibres, which are observed following chronic denervation, together with signs of primary myogenic defects, such as necrotic myofibers as well as myofibers with centrally located nuclei. ${ }^{16} 17$ Androgen-dependent impairment of myogenesis was observed in primary myoblasts isolated from patients with SBMA, although these cells proliferate and differentiate normally. ${ }^{18}$ Neurogenic and myopathic processes occur in SBMA mouse models. ${ }^{19} 20$ Notably, muscle atrophy foreshadows spinal cord pathology in knock-in SBMA mice, underscoring the primary role of skeletal muscles in SBMA pathogenesis. ${ }^{21}$ Tissue-specific expression of mutant AR in mice revealed that the sole expression of polyQ-AR in muscle leads to a phenotype that resembles SBMA, ${ }^{22}$ whereas expression in peripheral tissues but skeletal muscle prevents development of disease manifestations. ${ }^{23} 24$ Of note, SBMA manifestations in transgenic mice can be ameliorated by insulin-like growth factor 1 treatment or overexpression in muscle, which promotes AR degradation through phosphorylation by Akt. ${ }^{19}{ }^{25}$ While the degree of muscle atrophy seems to be directly correlated to the expression levels of AR, how polyQ-AR causes muscular atrophy is still largely unknown. Muscular fibres are classified into type I slow-oxidative, type IIa fast-oxidative and type IIb fast-glycolytic fibres. Typically, muscles contain all types of fibres innervated by their corresponding motor neurons. However, the ratio between slow and fast fibre types varies between muscles, so as to suit each muscle's function. Muscles of SBMA subjects demonstrate glycolytic-to-oxidative switching over the course of the disease. ${ }^{26}$ Remarkably, disease progression in AR113Q mice also results in a progressive shift from glycolytic to oxidative fibres, which precedes muscle atrophy and may be due to 
the preferential vulnerability of glycolytic fibres with respect to oxidative fibres. ${ }^{27}$ Furthermore, lipidomic analyses revealed an altered lipid composition in the muscle of knock-in mice compared with control animals. This metabolic alteration in muscle was associated with altered protein homeostasis, with concurrent activation of pathways of protein synthesis and degradation. ${ }^{27}$

These observations obtained in SBMA mice support the idea that skeletal muscle primarily degenerates, thereby contributing to motor neuron dysfunction and loss.

\section{SENSORY NEUROPATHY}

Along with muscular weakness, patients with SBMA may often complain of sensory disturbances, including numbness or tingling, and they mainly concerned the distal part of the lower limbs. ${ }^{28} 29$ In most cases, neurological examination reveals a slight vibration sense disorder without any abnormalities of the other sensory modalities. ${ }^{30}$ Conversely, neurophysiological studies have consistently detected peripheral low or absent sensory action potentials (SAPs) in the majority of patients, even when motor nerve conduction studies are within the normal range. ${ }^{31-33}$ Histopathology studies showed atrophy of either the dorsal root ganglion (DRG) neurons or the large myelinated sensory axons in the peripheral nervous system, thus leading to a definition of a primary SBMA sensory neuronopathy. ${ }^{34}$ Furthermore, nuclear and cytoplasmic accumulation of mutant AR has been observed in DRG neurons where it appears related to the CAG repeats length. ${ }^{1231}$ These pathological changes occur in patients with SBMA regardless of the disease duration, clinical severity and presence or absence of diabetes mellitus, indicating that sensory nerve involvement is primarily involved in this disease. ${ }^{34}$ Interestingly, Sobue and colleagues found SAPs to be decreased in cases with shorter CAG repeats, ${ }^{31}$ a finding not later confirmed in a larger cohort of Chinese patients with SBMA. ${ }^{33}$ Recent studies have identified a negative correlation between the expansion repeat size and the compound muscle action potential but not SAP amplitudes. ${ }^{35}$ On the other hand, the involvement of small myelinated and unmyelinated fibres demonstrated by means of skin biopsy ${ }^{36}$ could explain the presence of neuropathic pain reported by some patients. Lastly, a neurophysiological assessment of trigeminal reflexes, including the Blink reflex and the jaw-jerk, has suggested a trigeminal ganglionopathy. ${ }^{37}$

\section{AUTONOMIC DYSFUNCTION}

Patients with SBMA do not usually report symptoms suggestive of autonomic dysfunctions. However, in a study carried out in five SBMA subjects and investigating autonomic cardiovascular functions by a series of autonomic function tests, a subclinical involvement of both sympathetic and parasympathetic systems was observed. ${ }^{38}$ Previously, autonomic skin denervation with impaired sweat testing was reported in two patients. ${ }^{36}$ These findings are in line with the nuclear and cytoplasmic accumulation of expanded AR in neural autonomic regions, including hypothalamus, periaqueductal grey, nucleus ambiguus, spinal intermediolateral nucleus and sympathetic ganglia. ${ }^{12}$ Recently, a single patient with SBMA carrying 68 CAG repeats has been reported to have autonomic disturbances such as reduced sweating and difficulties in ejaculation. ${ }^{39}$

\section{CENTRAL NERVOUS SYSTEM INVOLVEMENT}

Recent studies have described subtle brain involvement in patients with SBMA. Significantly altered motor-evoked potential parameters were reporter in limb and cranial muscles in two siblings with clinical presentation and genetic testing consistent with diagnosis of SBMA. ${ }^{40}$ Analyses of white matter impairment using diffusion tensor imaging have revealed widespread alterations within the motor system, with a predominant deterioration of the corticospinal tract in patients with SBMA. ${ }^{41}$ Altogether these studies suggest subclinical involvement of the upper motor neuron tract in the disease.

Moreover, glucose hypometabolism in frontal areas of the cerebrum was detected in 10 patients with SBMA who underwent (18)F-fluorodeoxyglucose-positron emission tomography studies. ${ }^{42}$ Interestingly, neuropsychological tests administered to 20 patients with SBMA and to age-matched and education-matched controls showed distinctive deficits in mentalising, as assessed with the Faux Pas Test, although in the context of unimpaired neuropsychological performances. ${ }^{43}$

\section{METABOLIC FEATURES}

Evidence of metabolic dysfunction has been identified from several studies of patients with SBMA, including changes in the levels of glucose, dyslipidaemia and cholesterol. ${ }^{32} 334445 \mathrm{An}$ evaluation of biochemical profiles from a study of 57 American patients found that a majority had elevation of cholesterol and low-density lipoprotein (LDL) above the recommended levels. ${ }^{32}$ Similar features were identified in a large Italian cohort of 73 patients evaluated by Querin et al. ${ }^{44}$ Milder increases in cholesterol and LDL were detected in a Chinese population, although $>60 \%$ of those evaluated had hypertriglyceridaemia. ${ }^{33} \mathrm{~A}$ study of 55 Japanese patients found a significant increase in total cholesterol levels compared with controls. ${ }^{45}$ The relationship between cholesterol and LDL elevations with other features of the disease and lifestyle changes is not clear. Interestingly, disruptions in glucose metabolism and insulin resistance have also been reported in the disease. Although serum levels of glucose and HbA1c were found to be reduced in SBMA by Nakatsuji et al, the homeostasis model assessment of insulin resistance (HOMAIR) was significantly elevated in those with the disease compared with controls. ${ }^{45}$ Multivariate analysis identified the variables of body mass index (BMI), subcutaneous fat area, visceral fat area and testosterone as significant determinants of HOMA-IR. ${ }^{45}$ This study provides evidence of increased insulin resistance in SBMA, and the authors identify correlations between HOMA-IR and other motor features such as the revised ALS functional rating scale, Limb Norris Score and modified quantitative myasthenia gravis score. Analysis of skeletal muscle tissue and skin fibroblast cells shows reduced protein levels of insulin receptor $\beta$ subunit and insulin receptor substrate 1 in the SBMA samples, which may be contributing to the state of insulin resistance. It is not clear if the insulin resistance contributes to the neurodegeneration or is a consequence of the myopathic pathology. Dysfunction in other organ systems such as the liver may also be contributing to the changes in metabolism. Evidence of non-alcoholic steatohepatitis (NASH), despite normal BMI, was detected by magnetic resonance spectroscopy and further confirmed by liver biopsies in nearly all of the participants with SBMA evaluated in a recent study. ${ }^{46}$ This evidence, together with difference in gene expression pathways involved in fatty liver disease between patients with SBMA and those with typical NASH, suggests a direct association with the underlying disease. ${ }^{46}$

\section{BIOCHEMICAL AND HORMONAL FEATURES}

Several abnormalities in serum biochemical measurements have been previously reported in patients with SBMA including 
increases in CK, aspartate aminotransferase, alanine aminotransferase and lactate dehydrogenase. The elevation of these markers is indicative of the skeletal muscle pathology in the disease. Although these measurements are frequently abnormal, their levels have not been found to correlate with functional parameters of the disease. ${ }^{32}$ Low levels of creatinine have also been identified and correlate with functional measurements such as grip strength and the 6 min walk distance. ${ }^{47}$ The creatinine measurements may be a useful marker for tracking disease progression as these levels have been known to be associated with muscle mass. Serum creatinine levels have also been found to be lower in SBMA than patients with ALS who have similar muscle mass loss. ${ }^{48}$ Evaluation of the skeletal muscle from autopsy specimens identified decreased expression of the creatine transporter SLC6A8, providing a potential mechanism for the reduction in creatine levels in addition to the neurogenic aetiology. ${ }^{48}$ Measurements of the ligand for the AR, testosterone and its more potent derivative DHT have also been investigated in patients with SBMA. ${ }^{42} 33{ }^{44}$ Similarly to mice expressing polyQ-AR, which display decreased fertility, testicular atrophy and partial androgen insensitivity, ${ }^{21}$ patients frequently experience features of androgen loss of function including gynaecomastia, sexual dysfunction and testicular atrophy. Although most patients have androgen levels that fall within the normal reference range, some fall either above or below this reference range. ${ }^{32} 334477 \mathrm{An}$ evaluation of the androgen sensitivity index (testosterone levels multiplied by luteinizing hormone) found that about one-third of patients had increased index values, ${ }^{44}$ indicating a state of androgen resistance. Androstenedione, an intermediate in the synthesis of testosterone, was found to be reduced in another group of patients with SBMA. ${ }^{32}$ Multivariate regression analysis has identified that total testosterone levels positively correlate with quality of life as measured by the SF-36v2 questionnaire, muscle strength and activities of daily living. These findings suggest that in addition to activating AR toxicity, androgens may also be yielding potential anabolic-strengthening effects.

\section{CARDIAC ABNORMALITIES}

Although primary muscle involvement is a clear feature of SBMA, no detectable morphological and structural abnormalities appear in the myocardium, differently from numerous genetic myopathies. The typical nuclear AR accumulation, which is found in immunohistochemistry across numerous tissues, is also present in the myocardium and has potentially been linked to rhythm abnormalities which have been described in a significant proportion of SBMA sufferers. Araki et al first described ST alterations in ECGs of patients with SBMA, with Brugada syndrome-like alterations being the most common and found in $12 \%$ of cases. ${ }^{49}$ These abnormalities have been subsequently validated by other groups in different populations, although at lower frequency. ${ }^{44}$ Brugada syndrome can lead to sudden death, and screening of these abnormalities should become routine in patients with SBMA. Importantly, these abnormalities can be detected with ECG, and a simple modification in the conventional lead position significantly improves the detection of this defect and is recommended. ${ }^{50}$

\section{URINARY INVOLVEMENT}

The genitourinary tract involvement is among the previously unrecognised non-neuromuscular features of SBMA. Querin et al investigated a cohort of 71 patients with SBMA using the International Prostate Symptom Score (IPSS), followed by urodynamic and ultrasound investigations, when abnormalities in IPSS were found. ${ }^{44}$ Forty per cent of cases were either moderately or severely symptomatic for lower urinary tract symptoms (LUTS). The most prevalent finding was that of a bladder outlet obstruction in the absence of any indication for benign prostatic hyperplasia from biochemical and ultrasound investigations. It is not currently known whether LUTS are due to androgen insensitivity or a toxic AR expansion gain of function. It is nonetheless intriguing that knock-in mice carrying the AR expansion die due to alterations in the neuromuscular bladder axis, which determine acute urinary retention in the absence of a physical obstruction, ${ }^{21}$ thus further supporting the direct mechanistic link between the AR expansion and urinary dysfunction.

\section{CONCLUSIONS}

In SBMA, as in other genetic conditions caused by mutations in ubiquitously expressed genes, the clinical picture is the result of a complex interplay between differentially affected tissues, which struggle to cooperate to maintain homeostasis.

Extra-motor neuron features in SBMA, such as primary muscle atrophy or hormonal abnormalities, are emerging as clinically highly impactful in patients' quality of life and disease progression. Their thorough investigations are proving critical for a number of reasons. First, they may provide important insights into common mechanisms of pathogenesis. Second, the peripheral abnormalities may offer the opportunity for direct functional assessments and repetitive samplings, therefore representing potentially exploitable biomarkers to track disease progression and/or response to therapy. Lastly, disentangling the underlying molecular mechanisms of this highly integrated inter-tissues cross-talk may offer unparalleled opportunities for therapeutic interventions in the near future.

Contributors RM and CR: substantial contributions to the conception or design of the work. RM, GS, CG, PF, EZ, MP, and CR: drafting the work or revising it critically for important intellectual content and final approval of the version to be published.

Competing interests None declared.

Provenance and peer review Commissioned; externally peer reviewed.

Open access This is an open access article distributed in accordance with the Creative Commons Attribution Non Commercial (CC BY-NC 4.0) license, which permits others to distribute, remix, adapt, build upon this work non-commercially, and license their derivative works on different terms, provided the original work is properly cited and the use is non-commercial. See: http://creativecommons.org/ licenses/by-nc/4.0/

(c) Article author(s) (or their employer(s) unless otherwise stated in the text of the article) 2018. All rights reserved. No commercial use is permitted unless otherwise expressly granted.

\section{REFERENCES}

1 La Spada AR, Wilson EM, Lubahn DB, et al. Androgen receptor gene mutations in $X$-linked spinal and bulbar muscular atrophy. Nature 1991;352:77-9.

2 Rhodes LE, Freeman BK, Auh S, et al. Clinical features of spinal and bulbar muscular atrophy. Brain 2009;132:3242-51.

3 Sumner CJ, Fischbeck KH. Jaw drop in Kennedy's disease. Neurology 2002;59:1471-2.

4 Atsuta $\mathrm{N}$, Watanabe $\mathrm{H}$, Ito $\mathrm{M}$, et al. Natural history of spinal and bulbar muscular atrophy (SBMA): a study of 223 Japanese patients. Brain 2006;129(Pt 6):1446-55.

5 Fernández-Rhodes LE, Kokkinis AD, White MJ, et al. Efficacy and safety of dutasteride in patients with spinal and bulbar muscular atrophy: a randomised placebo-controlled trial. Lancet Neurol 2011;10:140-7.

6 Mangelsdorf DJ, Thummel C, Beato M, et al. The nuclear receptor superfamily: the second decade. Cell 1995:83:835-9.

7 Brinkmann AO. Molecular basis of androgen insensitivity. Mol Cell Endocrinol 2001;179:105-9.

8 Pennuto M, Rinaldi C. From gene to therapy in spinal and bulbar muscular atrophy: are we there yet? Mol Cell Endocrinol 2017;S0303-7207:30359-3.

9 Poletti A. The polyglutamine tract of androgen receptor: from functions to dysfunctions in motor neurons. Front Neuroendocrinol 2004;25:1-26. 
10 Li M, Chevalier-Larsen ES, Merry DE, et al. Soluble androgen receptor oligomers underlie pathology in a mouse model of spinobulbar muscular atrophy. J Biol Chem 2007;282:3157-64.

11 Rusmini P, Sau D, Crippa V, et al. Aggregation and proteasome: the case of elongated polyglutamine aggregation in spinal and bulbar muscular atrophy. Neurobio/ Aging 2007;28:1099-111.

12 Adachi H, Katsuno M, Minamiyama M, et al. Widespread nuclear and cytoplasmic accumulation of mutant androgen receptor in SBMA patients. Brain 2005; 128:659-70

13 Tanaka K, Matsuda N. Proteostasis and neurodegeneration: the roles of proteasomal degradation and autophagy. Biochim Biophys Acta 2014;1843:197-204.

14 Rinaldi C, Mäger I, Wood MJ. Proteostasis and Diseases of the Motor Unit. Front Mol Neurosci 2016;9:164

15 Lee JH, Shin JH, Park KP, et al. Phenotypic variability in Kennedy's disease: implication of the early diagnostic features. Acta Neurol Scand 2005;112:57-63.

16 Chahin N, Sorenson EJ. Serum creatine kinase levels in spinobulbar muscular atrophy and amyotrophic lateral sclerosis. Muscle Nerve 2009;40:126-9.

17 Sorarù G, D'Ascenzo C, Polo A, et al. Spinal and bulbar muscular atrophy: skeletal muscle pathology in male patients and heterozygous females. J Neurol $\mathrm{SCi}$ 2008;264:100-5.

18 Malena A, Pennuto M, Tezze C, et al. Androgen-dependent impairment of myogenesis in spinal and bulbar muscular atrophy. Acta Neuropathol 2013;126:109-21.

19 Palazzolo I, Stack C, Kong L, et al. Overexpression of IGF-1 in muscle attenuates disease in a mouse model of spinal and bulbar muscular atrophy. Neuron 2009;63:316-28.

20 Sambataro F, Pennuto M. Cell-autonomous and non-cell-autonomous toxicity in polyglutamine diseases. Prog Neurobiol 2012;97:152-72.

21 Yu Z, Dadgar N, Albertelli M, et al. Androgen-dependent pathology demonstrates myopathic contribution to the Kennedy disease phenotype in a mouse knock-in model. J Clin Invest 2006;116:2663-72.

22 Monks DA, Johansen JA, Mo K, et al. Overexpression of wild-type androgen receptor in muscle recapitulates polyglutamine disease. Proc Natl Acad Sci U S A 2007;104:18259-64.

23 Cortes CJ, Ling SC, Guo LT, et al. Muscle expression of mutant androgen receptor accounts for systemic and motor neuron disease phenotypes in spinal and bulbar muscular atrophy. Neuron 2014;82:295-307.

24 Lieberman AP, Yu Z, Murray S, et al. Peripheral androgen receptor gene suppression rescues disease in mouse models of spinal and bulbar muscular atrophy. Cell Rep 2014;7:774-84.

25 Rinaldi C, Bott L, et al. Insulinlike growth factor (IGF)-1 administration ameliorates disease manifestations in a mouse model of spinal and bulbar muscular atrophy. $\mathrm{Mol}$ Med 2012;18:1-8.

26 Yamada S, Hashizume A, Hijikata Y, et al. Decreased peak expiratory flow associated with muscle fiber-type switching in spinal and bulbar muscular atrophy. PLoS One 2016;11:e0168846.

27 Rocchi A, Milioto C, Parodi S, et al. Glycolytic-to-oxidative fiber-type switch and mTOR signaling activation are early-onset features of SBMA muscle modified by high-fat diet. Acta Neuropathol 2016:132:127-44.

28 Mariotti C, Castellotti B, Pareyson D, et al. Phenotypic manifestations associated with CAG-repeat expansion in the androgen receptor gene in male patients and heterozygous females: a clinical and molecular study of 30 families. Neuromuscul Disord 2000;10:391-7
29 Fratta P, Nirmalananthan N, Masset L, et al. Correlation of clinical and molecular features in spinal bulbar muscular atrophy. Neurology 2014;82:2077-84.

30 Sobue G, Hashizume Y, Mukai E, et al. X-linked recessive bulbospinal neuronopathy. A clinicopathological study. Brain 1989;112(Pt 1):209-32.

31 Suzuki K, Katsuno M, Banno H, et al. CAG repeat size correlates to electrophysiological motor and sensory phenotypes in SBMA. Brain 2008;131:229-39.

32 Rhodes LE, Freeman BK, Auh S, et al. Clinical features of spinal and bulbar muscular atrophy. Brain 2009;132(Pt 12):3242-51.

33 Ni W, Chen S, Qiao K, et al. Genotype-phenotype correlation in Chinese patients with spinal and bulbar muscular atrophy. PLoS One 2015;10:e0122279.

34 Li M, Sobue G, Doyu M, et al. Primary sensory neurons in X-linked recessive bulbospinal neuropathy: histopathology and androgen receptor gene expression. Muscle Nerve 1995;18:301-8.

$35 \mathrm{Kim} \mathrm{H}$, Lim YM, Lee EJ, et al. Correlation between the CAG repeat size and electrophysiological findings in patients with spinal and bulbar muscular atrophy. Muscle Nerve 2017 [Epub ahead of print 3 Oct 2017].

36 Manganelli F, lodice V, Provitera V, et al. Small-fiber involvement in spinobulbar muscular atrophy (Kennedy's disease). Muscle Nerve 2007;36:816-20.

37 Antonini G, Gragnani F, Romaniello A, et al. Sensory involvement in spinal-bulbar muscular atrophy (Kennedy's disease). Muscle Nerve 2000;23:252-8.

38 Rocchi C, Greco V, Urbani A, et al. Subclinical autonomic dysfunction in spinobulbar muscular atrophy (Kennedy disease). Muscle Nerve 2011;44:737-40.

39 Grunseich C, Kats IR, Bott LC, et al. Early onset and novel features in a spinal and bulbar muscular atrophy patient with a 68 CAG repeat. Neuromuscul Disord 2014;24:978-81.

40 Pachatz C, Terracciano C, Desiato MT, et al. Upper motor neuron involvement in X-linked recessive bulbospinal muscular atrophy. Clin Neurophysiol 2007;118:262-8

41 Unrath A, Müller HP, Riecker A, et al. Whole brain-based analysis of regional white matter tract alterations in rare motor neuron diseases by diffusion tensor imaging. Hum Brain Mapp 2010;31:1727-40.

42 Lai TH, Liu RS, Yang BH, et al. Cerebral involvement in spinal and bulbar muscular atrophy (Kennedy's disease): a pilot study of PET. J Neurol Sci 2013;335:139-44.

43 Di Rosa E, Sorarù G, Kleinbub JR, et al. Theory of mind, empathy and neuropsychological functioning in X-linked spinal and bulbar muscular atrophy: a controlled study of 20 patients. J Neurol 2015;262:394-401.

44 Querin G, Bertolin C, Da Re E, et al. Non-neural phenotype of spinal and bulbar muscular atrophy: results from a large cohort of Italian patients. J Neurol Neurosurg Psychiatry 2016;87:810-6.

45 Nakatsuji H, Araki A, Hashizume A, et al. Correlation of insulin resistance and motor function in spinal and bulbar muscular atrophy. J Neurol 2017;264:839-47.

46 Guber RD, Takyar V, Kokkinis A, et al. Nonalcoholic fatty liver disease in spinal and bulbar muscular atrophy. Neurology 2017;89:2481-90.

47 Hashizume A, Katsuno M, Banno H, et al. Longitudinal changes of outcome measures in spinal and bulbar muscular atrophy. Brain 2012;135:2838-48.

48 Hijikata Y, Katsuno M, Suzuki K, et al. Impaired muscle uptake of creatine in spinal and bulbar muscular atrophy. Ann Clin Trans/ Neurol 2016:3:537-46.

49 Araki A, Katsuno M, Suzuki K, et al. Brugada syndrome in spinal and bulbar muscular atrophy. Neurology 2014:82:1813-21.

50 Naseef A, Behr ER, Batchvarov VN. Electrocardiographic methods for diagnosis and risk stratification in the Brugada syndrome. J Saudi Heart Assoc 2015;27:96-108. 